\title{
SASAKIAN HYPERSURFACES OF THE GENERALIZED CONCIRCULAR RECURRENT KAHLERIAN MANIFOLD
}

\author{
Y. B. Maralabhavi* \& Hari Baskar R.**
}

\begin{abstract}
In this paper we consider a recurrent sasakian hypersurface of the generalized concircular recurrent Kahlerian manifold and determine some conditions on the vector fields used in the sasakion structure. Further we determine such conditions for $\varphi$ sasakian hypersurface also.
\end{abstract}

\section{Preliminaries}

Let $\mathrm{M}^{2 \mathrm{n}+2}$ be a $2 \mathrm{n}+2$ dimensional almost Hermitian manifold, with structure tensors $(J, G)$ and the Riemannian connection $\ddot{\nabla}$ such that $\mathrm{J}^{2}=-\mathrm{I}$ and $G(J X, J Y)=G(X, Y)$. An almost Hermitian manifold with $\tilde{\nabla} J=0$ is known as Kahlerian manifold. Suppose that $M^{2 n+1}$ is a $C^{\infty}$ hypersurface of $M^{2 n+2}$ with unit normal $\mathrm{N}$ and the induced metric $\mathrm{g}$. Thus if di denotes the differential of the imbedding $\mathrm{i}: \mathrm{M}^{2 \mathrm{n}+1} \rightarrow \mathrm{M}^{2 n+2}, \mathrm{X}$ a vector field on $M^{2 n+1}$, then $\tilde{X}$ is

* Department of Mathematics, Bangalore University, Bangalore, India.

** Department of Mathematics, Christ University, Bangalore, India. 
the extension on $M^{2 n+2}$ of $\tilde{X}$ and is such that $\tilde{X}$ restricted to $M^{2 n+1}$ under the imbedding is diX. Also let $\Theta=\left\{e_{i}\right\}, i=1,2, \ldots \ldots, 2 n+1$ be an orthonormal basis of the tangent space at any point of the manifold $M^{2 n+1}$ then $\bar{\Theta}=\left\{e_{i}, N\right\}$ $\mathrm{i}=1,2, \ldots \ldots, 2 \mathrm{n}+1$ is an orthonormal basis for the tangent space at any point on the manifold $M^{2 n+2}$. Hence

$$
G(\tilde{X}, \tilde{X})=g(X, Y), \quad G(N, N)=1, \quad G(\tilde{X}, N)=0
$$

and its Riemannian connection $\nabla$ is governed by Gauss-Weingarten equations

$$
\tilde{\nabla}_{\tilde{\mathrm{X}}} \tilde{\mathrm{Y}}=\left(\nabla_{\mathrm{X}} \mathrm{Y}\right)+\mathrm{h}(\mathrm{X}, \mathrm{Y}) \mathrm{N}, \quad \tilde{\nabla}_{\tilde{\mathrm{X}}^{\mathrm{N}}} \mathrm{N}=-\left(\tilde{\mathrm{H}^{\prime} \mathrm{X}}\right)
$$

where $h$ denotes the second fundamental form and $\mathrm{H}^{\prime}$ the corresponding Weingarten map. Also the submanifold $\mathrm{M}^{2 \mathrm{n}+1}$ inherits an almost contact metric structure $(\varphi, \xi, \eta, g)[1][2]$ given by

$$
J \tilde{X}=(\tilde{\varphi} X)+\eta(X) N, \quad J N=-\bar{\xi}
$$

Then (1.1), (1.2) and (1.3) lead to the following conditions in $M^{2 n+1}$ : $\varphi^{2}=-I+\eta \otimes \xi, \quad \eta \circ \varphi=0, \quad \varphi \xi=0, \quad \eta(\xi)=1$,

$$
\mathrm{g}(\varphi \mathrm{X}, \varphi \mathrm{Y})=\mathrm{g}(\mathrm{X}, \mathrm{Y})-\eta(X) \eta(\mathrm{X}), \quad \eta(X)=\mathrm{g}(\mathrm{X}, \xi) .
$$

An almost contáct metric structure $(\varphi, \xi, \eta, g)$ is Sasakian if and only if

$$
\left(\nabla_{X} \varphi\right) Y=g(X, Y) \xi-\eta(Y) X
$$

If $\mathrm{K}$ is the curvature tensor of type $(0,4)$ and $S$ is the Ricci tensor of type $(0,2)$ in the sasakian manifold $\mathrm{M}^{2 \mathrm{n}+1}$, then the following conditions [ 1$]$ hold in a sasakian manifold 


$$
S(X, \xi)=2 n \eta(X)
$$

$g(K(\xi, X) Y, \xi)=g(X, Y)-\eta(X) \eta(X)$

$\mathrm{K}(\xi, \mathrm{X}) \xi=-\mathrm{X}+\eta(\mathrm{X}) \xi$

$g(K(X, Y) \xi, Z)=g(X, Z) \eta(Y)-g(Y, Z) \eta(X)$

$\left(\nabla_{X} \phi\right)(Y)=R(\xi, X) Y$

If $\Omega$ is the 2-form on $\mathrm{M}^{2 \mathrm{n}+1}$ defined by

$$
\Omega(X, Y)=g(X ; \varphi Y)=-g(\varphi X, Y)=-\Omega(Y, X)
$$

then from (1.5), we get

$$
\left(\nabla_{X} \eta\right)(Y)=g(X, \varphi Y)
$$

If $\tilde{K}$ the curvature tensor in the Kahlerian manifold $\mathrm{M}^{2 \mathrm{n}+2}$, then we have the following well known Gauss-Codazzi equations

$$
\begin{aligned}
\mathrm{K}(\mathrm{X}, \mathrm{X}, \mathrm{Z}, \mathrm{W})=\tilde{\mathrm{K}}(\tilde{\mathrm{X}}, \tilde{\mathrm{Y}}, \tilde{\mathrm{Z}}, \tilde{\mathrm{W}}) & \\
& +\mathrm{h}(\mathrm{X}, \mathrm{W}) \mathrm{h}(\mathrm{Y}, \mathrm{Z})-\mathrm{h}(\mathrm{X}, \mathrm{Z}) \mathrm{h}(\mathrm{Y}, \mathrm{W})
\end{aligned}
$$

$\tilde{\mathrm{K}}(\tilde{X}, \tilde{Y}, \tilde{Z}, N)=\left(\nabla_{X} h\right)(\mathrm{Y}, Z)-\left(\nabla_{Y} h\right)(X, Z)$

where $\tilde{K}(\tilde{X}, \tilde{Y}, \vec{Z}, \tilde{W})$ and $K(X, Y, Z, W)$ are given by $G(\tilde{K}(\tilde{X}, \vec{Y}) \tilde{Z}, \vec{W})$ and $\mathrm{g}(\mathrm{K}(\mathrm{X}, \mathrm{Y}) \mathrm{Z}, \mathrm{W})$ respectively.

A Kahlerian manifold $\mathrm{M}^{2 \mathrm{n}+2}$ is said to be a generalized concircular recurrent manifold [6] if there exists a non-zero 1 -forms $\tilde{A}$ and $\tilde{B}$ such that

$$
\left(\tilde{\nabla}_{\tilde{U}} \tilde{\mathrm{C}}\right)(\tilde{\mathrm{X}}, \tilde{\mathrm{Y}}, \tilde{\mathrm{Z}}, \overrightarrow{\mathrm{W}})=\tilde{\mathrm{A}}(\tilde{\mathrm{U}}) \tilde{\mathrm{C}}(\tilde{\mathrm{X}}, \tilde{\mathrm{Y}}, \overrightarrow{\mathrm{Z}}, \overrightarrow{\mathrm{W}})+\dot{\mathrm{B}}(\overrightarrow{\mathrm{U}}) \overrightarrow{\mathrm{F}}(\overrightarrow{\mathrm{X}}, \overline{\overline{\mathrm{Y}}}, \overline{\underline{Z}}, \overline{\mathrm{W}})
$$


for arbitrany vector fields $\tilde{X}, \tilde{Y}, \tilde{Z}, \tilde{W}$ and $\tilde{U}$ on $M^{2 n+2}$, where

$$
\tilde{\mathrm{C}}(\tilde{\mathrm{X}}, \tilde{\mathrm{Y}}, \tilde{\mathrm{Z}}, \tilde{\mathrm{W}})=\tilde{\mathrm{K}}(\tilde{\mathrm{X}}, \tilde{\mathrm{Y}}, \tilde{\mathrm{Z}}, \tilde{\mathrm{W}})-\frac{\mathrm{R}}{(2 \mathrm{n}+1)(2 \mathrm{n}+2)} \tilde{\mathrm{F}}(\tilde{\mathrm{X}}, \underline{\overline{\mathrm{Y}}}, \underline{\underline{Z}}, \overline{\vec{W}})
$$

and

$$
\tilde{\mathrm{F}}(\tilde{\mathrm{X}}, \overline{\tilde{Y}}, \underline{\underline{Z}}, \overline{\tilde{W}})=\mathrm{G}(\tilde{\mathrm{X}}, \tilde{\mathrm{W}}) \mathrm{G}(\tilde{\mathrm{Z}}, \tilde{\mathrm{Y}})-\mathrm{G}(\tilde{\mathrm{X}}, \tilde{\mathrm{Z}}) \mathrm{G}(\tilde{\mathrm{Y}}, \tilde{\mathrm{W}})
$$

with $\tilde{A}(\tilde{U})=G\left(\tilde{U}, \tilde{\rho}^{\prime}\right)$ and $\tilde{B}(\tilde{U})=G\left(\tilde{U}, \tilde{\rho}^{\prime \prime}\right)$ for some vectorfields $\tilde{\rho}^{\prime}$, $\tilde{\rho}^{\prime \prime}$. (Note: In (1.18), the bars above $\tilde{Y}$ and $\tilde{W}$ indicate that they are swapped to get the first term from $G(\tilde{X}, \tilde{Y}) G(\tilde{Z}, \tilde{W})$ and the bars below $\tilde{Y}$ and $\tilde{Z}$ indicates that they are swapped to get the second term from $G(\tilde{X}, \tilde{Y}) G(\tilde{Z}, \tilde{W})$. If the second fundamental tensor $\mathrm{h}(\mathrm{X}, \mathrm{Y})$ satisfies the condition [4]

$$
h(X, Y)=g(X, Y)+\mu \eta(X) \eta(Y)
$$

then $\mathrm{M}^{2 \mathrm{n}+1}$ is called a c-umbilical hypersurface. Seiichi Yamaguchi [4] has proved that an immersed hypersurface in Kahlerian manifold is sasakian if and only if it is cumbilical, with

$\mu=(2 n+1)(H-1)$

in (1.19), where $H$ is the mean curvature. If $H$ is a constant then $\nabla \mu=0$ and the immersed hypersurface is called as the CMC hypersurface.

\section{Recurrent Sasakian Hypersurfaces:}

Definition 2.1 A Sasakian manifold is said to be a Recurrent manifold [3] if there exists a non-zero 1 -form $A(U)$ such that

$$
\left(\nabla_{U} \mathrm{~K}\right)(\mathrm{X}, \mathrm{Y}, \mathrm{Z}, \mathrm{W})=\mathrm{A}(\mathrm{U}) \mathrm{K}(\mathrm{X}, \mathrm{Y}, \mathrm{Z}, \mathrm{W})
$$


Where $\mathrm{X}, \mathrm{Y}, \mathrm{Z}, \mathrm{W}$ and $\mathrm{U}$ are the arbitrary vector fields and $\mathrm{A}(\mathrm{U})=\mathrm{g}\left(\mathrm{U}, \rho^{\prime}\right)=\mathrm{G}\left(\tilde{U}, \vec{\rho}^{\prime}\right)=\overrightarrow{\mathrm{A}}(\tilde{U})$, for some vector field $\rho^{\prime}$.

Theorem 2.2 Suppose $M^{2 n+1}$ is a recurrent sasakian hypersurface of a generalized concircular recurrent Kahlerian manifold $\mathrm{M}^{2 \mathrm{n}+2}$, then $\xi$ is orthogonal to the vector field $\rho^{\prime \prime}-\lambda \rho^{\prime}$ if and only if $\xi[R]=A(\xi) R-4(n+1) \xi[\mu]$, where $\lambda=1+(2 \mu /(2 n+1))$ and $\rho^{\prime}$ and $\rho^{\prime \prime}$ are the vector fields associated with the one forms $A$ and $B$.

Proof By applying $\nabla_{U}$ to (1.17) and using (1.1), (1.2), (1.4), (1.5), (1.12), $(1.13),(1.14),(1.15),(1.16),(1.17),(1.18)$ and $(1.19)$ we get

$$
\begin{aligned}
& \left(\nabla_{\mathrm{U}} \mathrm{K}\right)(\mathrm{X}, \mathrm{Y}, \mathrm{Z}, \mathrm{W})=\mathrm{A}(\mathrm{U}) \mathrm{K}(\mathrm{X}, \mathrm{Y}, \mathrm{Z}, \mathrm{W}) \\
& +\left\{B(U)-A(U)+\frac{\tilde{U}[\tilde{R}]-R A(U)}{(2 n+1)(2 n+2)}\right\} F(X, \bar{Y}, \underline{Z}, \bar{W}) \\
& +\{U[\mu]-A(U)\}\{n(X) F(\xi, \underline{\bar{Y}}, \underline{Z}, \bar{W}) \\
& +\eta(\mathrm{Y}) \mathrm{F}(\mathrm{X}, \vec{\xi}, \underline{\mathrm{Z}}, \overline{\mathrm{W}})\} \\
& +\mathrm{g}(\mathrm{U}, \mathrm{X})\{(\mathrm{W}[\mu] \eta(\mathrm{Z})-\mathrm{Z}[\mu] \eta(W)) \eta(\mathrm{Y})\} \\
& +\mathrm{g}(\mathrm{U}, \mathrm{X})\{(\mathrm{Z}[\mu] \eta(\mathrm{W})-\mathrm{W}[\mu] \eta(\mathrm{Z})) \eta(\mathrm{X})\} \\
& \operatorname{tg}(U, Z)\{(Y[\mu] \eta(X)-X[\mu] \eta(X)) \eta(W)\} \\
& +\mathrm{g}(\mathrm{U}, \mathrm{W})\{(\mathrm{X}[\mu] \eta(\mathrm{Y})-\mathrm{X}[\mu] \eta(\mathrm{X})) \eta(\mathrm{Z})\} \\
& +\mu \mathrm{g}(\mathrm{U}, \mathrm{X})(2 \Omega(\mathrm{W}, \mathrm{Z}) \eta(\mathrm{Y})-\mathrm{F}(\xi, \overline{\varphi \mathrm{Y}}, \mathrm{Z}, \overline{\mathrm{W}})) \\
& +\mu \mathrm{g}(\mathrm{U}, \mathrm{Y})(2 \Omega(\mathrm{Z}, \mathrm{W}) \eta(\mathrm{X})-\mathrm{F}(\varphi \mathrm{X}, \overline{\underline{\xi}}, \underline{\mathrm{Z}}, \overline{\mathrm{W}})) \\
& +\mu \mathrm{g}(\mathrm{U}, \mathrm{Z})(2 \Omega(\mathrm{Y}, \mathrm{X}) \eta(\mathrm{W})-\mathrm{F}(\mathrm{X}, \overline{\mathrm{Y}}, \underline{\xi}, \bar{\varphi} \overline{\mathrm{W}})) \\
& +\mu \mathrm{g}(\mathrm{U}, \mathrm{W})(2 \Omega(\mathrm{X}, \mathrm{Y}) \eta(Z)-\mathrm{F}(\mathrm{X}, \underline{\bar{Y}}, \underline{\varphi Z}, \bar{\xi})) \\
& +\mu(\Omega(\mathrm{U}, \mathrm{X}) \mathrm{F}(\xi, \overline{\mathrm{Y}}, \underline{\mathrm{Z}}, \overline{\mathrm{W}})+\Omega(\mathrm{U}, \mathrm{Y}) \mathrm{F}(\mathrm{X}, \bar{\xi}, \underline{\mathrm{Z}}, \overline{\mathrm{W}})) \\
& +\mu(\Omega(\mathrm{U}, \mathrm{Z}) \mathrm{F}(\mathrm{X}, \underline{\bar{Y}}, \underline{\xi}, \overline{\mathrm{W}})+\Omega(\mathrm{U}, \mathrm{W}) \mathrm{F}(\mathrm{X}, \underline{\bar{Y}}, \underline{\mathrm{Z}}, \bar{\xi}))
\end{aligned}
$$


Since $M^{2 n+1}$ is recurrent, we use (2.1) in (2.2). Then by replacing $U$ by $\xi$ and choosing $X=W=e_{i}, Y=Z=e_{j}$, and taking summation over $i, j$ $1 \leq i, j \leq 2 n+1$, we get

$$
\begin{aligned}
\tilde{\xi}[R]= & A(\xi) R \\
& +2(n+1)[(2(n+\mu)+1) A(\xi)-(2 n+1) B(\xi)] \\
& -4(n+1) \xi[\mu]
\end{aligned}
$$

and hence the theorem.

By virtue of theorem 2.2 and (1.20), we have

Corollary 2.3 Suppose $\mathrm{M}^{2 \mathrm{n}+1}$ is a recurrent sasakian hypersurface of a generalized concircular recurrent Kahlerian manifold $M^{2 n+2}$. Then $M^{2 n+1}$ is $C M C$ if and only if $\tilde{\xi}[R]=R \tilde{A}(\tilde{\xi})$.

Corollary 2.4 Suppose $M^{2 n+1}$ is a recurrent sasakian $C M C$ hypersurface of a generalized concircular recurrent Kahlerian manifold $M^{2 n+2}$, then $M^{2 n+2}$ is flat if and only if $R=0$.

\section{3. $\varphi$-recurrent Sasakian Hypersurfaces:}

Definition 3.1 A Sasakian manifold is said to be $\varphi$ - recurrent manifold [5] if there exists a non-zero 1 -form $A$ such that

$$
\varphi^{2}\left(\left(\nabla_{U} K\right)(X, Y, Z, W)=A(U) K(X, Y, Z, W)\right.
$$

for arbitrary vectorfields $\mathrm{X}, \mathrm{Y}, \mathrm{Z}, \mathrm{W}$ and $\mathrm{U}$ where $\mathrm{K}$ is the curvature tensor in Sasakian manifold.

If a Sasakian manifold $M^{2 n+1}$ is a $\varphi$ - recurrent manifold, then the following relation holds [5] 
$\left(\nabla_{\mathrm{U}} \mathrm{K}\right)(X, Y, Z, W)=\{\mathrm{g}(Y, \mathrm{U}) \mathrm{g}(\varphi \mathrm{X}, \mathrm{Z})-\mathrm{g}(\mathrm{X}, \mathrm{U}) \mathrm{g}(\varphi \mathrm{Y}, \mathrm{Z})$

$$
\begin{array}{r}
-\mathrm{g}(\varphi \mathrm{K}(\mathrm{X}, \mathrm{Y}) \mathrm{U}, Z) \mathrm{g} g(\xi, W) \\
-\mathrm{A}(\mathrm{U}) \mathrm{K}(\mathrm{X}, \mathrm{Y}, \mathrm{Z} . \mathrm{W})
\end{array}
$$

Theorem 3.2 Suppose $M^{2 n+1}$ is a $\varphi$-recurrent sasakian hypersueneralized concircular recurrent Kahlerian manifold $\mathrm{M}^{2 \mathrm{n}+2}$. Then $\xi$ is orthogonal to the vector field $\rho^{\prime \prime}-\lambda \rho^{\prime}$ if and only if $\xi[R]=A(\xi) R-4(n+1) \xi[\mu]$, where $\lambda=1+\frac{2 \mu n-r}{n(2 n+1)}$ and $\rho^{\prime}, \rho^{\prime \prime}$ are the vector fields associated with the one forms $\mathrm{A}$ and $\mathrm{B}$.

Proof By applying $\nabla_{U}$ to $(1.12)$ and using $(1.1),(1.2),(1.4),(1.5),(1.7),(1.8)$, (1.9), (1.10), (1.11), (1.12), (1.13) and (1.14) we get the expression, same as (2:2). Since $M^{2 n+1}$ is $\varphi$-recurrent we use (3.2) in (2.2) to obtain.

\section{$2 \mathrm{~A}(\mathrm{U}) \mathrm{K}(\mathrm{X}, \mathrm{Y}, \mathrm{Z}, \mathrm{W})$}

$+A(U)\{[g(X, U) g(\varphi Y, U)-g(Y, U) g(\varphi X, Z)]$

$-\mathrm{K}(\mathrm{X}, \mathrm{Y}, \mathrm{U}, \varphi \mathrm{Z}) \mathrm{g}(\xi, \mathrm{W})$

$+\left\{B(U)-A(U)+\frac{\tilde{U}[\tilde{R}]-R A(U)}{(2 n+1)(2 n+2)}\right\} F(X, \overline{\underline{X}}, \underline{Z}, \bar{W})$

$+\{U[\mu]-A(U)\}\{\eta(X) F(\xi, \underline{Y}, \underline{Z}, \bar{W})+\eta(Y) F(X, \bar{\xi}, \underline{Z}, \bar{W})\}$

$+\mathrm{g}(\mathrm{U}, \mathrm{X})\{(\mathrm{W}[\mu] \eta(\mathrm{Z})-\mathrm{Z}[\mu] \eta(\mathrm{W})) \eta(\mathrm{Y})\}$

$+g(U, Y)\{(Z[\mu] \eta(W)-W[\mu] \eta(Z)) \eta(X)\}$

$+\mathrm{g}(\mathrm{U}, \mathrm{Z})\{(\mathrm{Y}[\mu] \eta(\mathrm{X})-\mathrm{X}[\mu] \eta(\mathrm{Y})) \eta(\mathrm{W})\}$

$+\mathrm{g}(U, W)\{(X[\mu] \eta(Y)-Y[\mu] \eta(X)) \eta(Z)\}$

$+\mu \mathrm{g}(\mathrm{U}, \mathrm{X})(2 \Omega(\mathrm{W}, \mathrm{Z}) \eta(\mathrm{Y})-\mathrm{F}(\xi, \overline{\underline{\varphi}}, \underline{\mathrm{Z}}, \overline{\mathrm{W}}))$

$+\mu \mathrm{g}(\mathrm{U}, \mathrm{Y})(2 \Omega(\mathrm{Z}, \mathrm{W}) \eta(\mathrm{X})-\mathrm{F}(\varphi \mathrm{X}, \bar{\xi}, \underline{Z}, \bar{W}))$

$+\mu \mathrm{g}(\mathrm{U}, \mathrm{Z})(2 \Omega(\mathrm{Y}, \mathrm{X}) \eta(\mathrm{W})-\mathrm{F}(\mathrm{X}, \underline{\bar{Y}}, \underline{\xi}, \overline{\varphi \mathrm{W}}))$

$+\mu \mathrm{g}(\mathrm{U}, \mathrm{W})(2 \Omega(\mathrm{X}, \mathrm{Y}) \eta(\mathrm{Z})-\mathrm{F}(\mathrm{X}, \underline{\overline{\mathrm{Y}}}, \underline{\varphi \mathrm{Z}}, \bar{\xi}))$

$+\mu(\Omega(\mathrm{U}, \mathrm{X}) \mathrm{F}(\xi, \underline{\bar{Y}}, \underline{Z}, \overline{\mathrm{W}})+\Omega(\mathrm{U}, \mathrm{X}) \mathrm{F}(\mathrm{X}, \overline{\bar{\xi}}, \underline{Z}, \overline{\mathrm{W}}))$

$+\mu(\Omega(U, Z) F(X, \bar{Y}, \underline{\xi}, \bar{W})+\Omega(U, W) F(X, \bar{Y}, \underline{\xi}, \bar{W}))=0$ 
Replacing $U$ by $\xi$, and choosing $X=W=e_{i}, Y=Z=e_{j}$ and taking the summation over $i, j, 1 \leq i, j \leq 2 n+1$, we get

$$
\begin{aligned}
\tilde{\xi}[R]= & A(\xi) R \\
& +2(n+1)\left[\left(2(n+\mu)-\frac{r}{n}\right) A(\xi)-(2 n+1) B(\xi)\right] \\
& -4(n+1) \xi[\mu]
\end{aligned}
$$

hence the theorem.

In [5] it has been proved that "A $\varphi$-recurrent sasakian manifold $\left(M^{2 n+1}, g\right)$, $\mathrm{n}>1$, is a space of constant curvature, provided that $X$ and $\mathrm{Y}$ are orthogonal to $\xi$ ".

Using the condition in the above theorem that $X$ and $\mathrm{Y}$ are orthogonal to $\xi$ in (3.3) and replacing $U$ by $\xi$, and choosing $X=W=e_{i}, Y=Z=e_{i}$, and taking summation over $i, j, 1 \leq i, j \leq 2 n+1$, we get

$$
\begin{aligned}
\tilde{\xi}[R]= & A(\xi) R \\
& +2(n+1)\left[\left(2 n+1-\frac{r}{n}\right) A(\xi)-(2 n+1) B(\xi)\right]
\end{aligned}
$$

Hence we have the theorem:

Theorem 3.4 Suppose $M^{2 n+1}$ is a $\varphi$ - recurrent sasakian space of constant curvature immersed in a generalized concircular recurrent Kahlerian manifold $\mathrm{M}^{2 \mathrm{n}+2}, \xi$ is orthogonal to $\rho^{\prime \prime}-\lambda \rho^{\prime}$ if and only if $\xi[R]=R A(\xi)$, where $\lambda=1-\frac{r}{n(2 n+1)}$ and $\rho^{\prime}, \rho^{\prime \prime}$ are the vector fields associated with the one forms $A$ and $B$.

By virtue of the theorem 3.2, theorem 3.4 and using $(1.20)$ we have: 
Corollary 3.5 Suppose $\mathrm{M}^{2 \mathrm{n}+1}$ is a $\varphi$ - recurrent sasakian space of constant curvature immersed in a generalized concircular recurrent Kahlerian manifold $\mathrm{M}^{2 \mathrm{n}+2}$. Then the mean curvature $\mathrm{H}=1$ in $\mathrm{M}^{2 \mathrm{n}+1}$.

Acknowledgement: The second author would like to acknowledge the support of Christ University administration for deputing him to Ph.D Programme.

\section{References}

1. D. E. Blair, Contact Manifolds in Riemannian Geometry, Lecture notes in Mathematics, Vol. 509, Springer Verlag, Berlin - New York, 1976.

2. David E Blair, Gerald D Ludden and Kentaro Yano, Induced Structures on Submanifolds, Kodai Math. Sem. Rep. 22(1970), 188-198.

3. Mileva Provanovic, On some hypersurfaces of a Recurrent Riemannian Space, Publications de L'Institut Mathematique, Nouvelle serietome 47 (61), 1990, 103-112.

4. Seiichi Yamaguchi, On a c-umbilical hypersuraface of a Kahlerian manifold with vanishing bochner curvature tensor, Tensor N S, Vol 20 (1969).

5. U.C.De, A.A. Shaikh, Sudipta Biswas, "On $\varphi$ - recurrent sasakian manifolds", Novi Sad J. Math, Vol.33, No.2, 2003, 43-48.

6. U.C.De AND D Kamilya,"On generalized concircular recurrent manifold, Bull. Cal. Math. Soc., 86, 69.72 (1994). 